\title{
Augmented down-up algebras and uniform posets
}

\author{
Paul Terwilliger*, Chalermpong Worawannotai \\ Department of Mathematics, University of Wisconsin \\ 480 Lincoln Drive, Madison, WI 53706-1388 USA
}

Received 9 July 2013, accepted 31 August 2013, published online 9 September 2013

\begin{abstract}
Motivated by the structure of the uniform posets we introduce the notion of an augmented down-up (or ADU) algebra. We discuss how ADU algebras are related to the down-up algebras defined by Benkart and Roby. For each ADU algebra we give two presentations by generators and relations. We also display a $\mathbb{Z}$-grading and a linear basis. In addition we show that the center is isomorphic to a polynomial algebra in two variables. We display seven families of uniform posets and show that each gives an ADU algebra module in a natural way. The main inspiration for the ADU algebra concept comes from the second author's thesis concerning a type of uniform poset constructed using a dual polar graph.
\end{abstract}

Keywords: Uniform poset, dual polar space, dual polar graph, down-up algebra.

Math. Subj. Class.: 06A07, 05E10, 17B37

\section{Introduction}

In [10] the first author introduced the notion of a uniform poset, and constructed eleven families of examples from the classical geometries. Among the examples are the polar spaces $\operatorname{Polar}_{b}(N, \epsilon)$ and the attenuated spaces $\mathrm{A}_{b}(N, M)$, as well as the posets $\operatorname{Alt}_{b}(N)$, $\operatorname{Her}_{q}(N)$, and $\operatorname{Quad}_{b}(N)$ associated with the alternating, Hermitean, and quadratic forms. Another example is Hemmeter's poset $\operatorname{Hem}_{b}(N)$. In [12, Proposition 26.4] the second author constructed a new family of uniform posets using the dual polar graphs. We denote these posets by $\operatorname{Polar}_{b}^{\text {top }}(N, \epsilon)$ and describe them in Section 5 below.

In [2] Benkart and Roby introduced the down-up algebras, and obtained modules for these algebras using $\operatorname{Alt}_{b}(N), \operatorname{Her}_{q}(N), \operatorname{Quad}_{b}(N)$, and $\operatorname{Hem}_{b}(N)$. A down-up algebra module is obtained from $\operatorname{Polar}_{b}^{\text {top }}(N, \epsilon)$ in a similar way. However, it appears that the downup algebra concept is not sufficiently robust to handle $\operatorname{Polar}_{b}(N, \epsilon)$ or $\mathrm{A}_{b}(N, M)$. The

\footnotetext{
${ }^{*}$ Corresponding author

E-mail addresses: terwilli@math.wisc.edu (Paul Terwilliger),worawann@math.wisc.edu (Chalermpong Worawannotai)
} 
same can be said for the generalized down-up algebras [5]. In the present paper we introduce a family of algebras called augmented down-up algebras, or ADU algebras for short. These algebras seem well suited to handle uniform posets. Indeed, we show that each of the uniform posets $\operatorname{Polar}_{b}(N, \epsilon), \mathrm{A}_{b}(N, M), \operatorname{Alt}_{b}(N), \operatorname{Her}_{q}(N), \operatorname{Quad}_{b}(N), \operatorname{Hem}_{b}(N)$, $\operatorname{Polar}_{b}^{\text {top }}(N, \epsilon)$ gives an ADU algebra module in a natural way.

The ADU algebras are related to the down-up algebras as follows. Given scalars $\alpha, \beta, \gamma$ the corresponding down-up algebra $A(\alpha, \beta, \gamma)$ is defined by generators $e, f$ and relations

$$
\begin{aligned}
& e^{2} f=\alpha e f e+\beta f e^{2}+\gamma e, \\
& e f^{2}=\alpha f e f+\beta f^{2} e+\gamma f .
\end{aligned}
$$

See [2, p. 308]. To turn this into an ADU algebra we make three adjustments as follows. Let $q$ denote a nonzero scalar that is not a root of unity. We first require

$$
\alpha=q^{-2 s}+q^{-2 t}, \quad \beta=-q^{-2 s-2 t}
$$

where $s, t$ are distinct integers. Secondly, we add two generators $k^{ \pm 1}$ such that $k e=q^{2} e k$ and $k f=q^{-2} f k$. Finally we reinterpret $\gamma$ as a Laurent polynomial in $k$ for which the coefficients of $k^{s}, k^{t}$ are zero.

From the above description the ADU algebras are reminiscent of the quantum univeral enveloping algebra $U_{q}\left(\mathfrak{s l}_{2}\right)$. To illuminate the difference between these algebras, consider their center. By [6, p. 27] the center of $U_{q}\left(\mathfrak{s l}_{2}\right)$ is isomorphic to a polynomial algebra in one variable. As we will see, the center of an ADU algebra is isomorphic to a polynomial algebra in two variables.

The results of the present paper are summarized as follows. We define two algebras by generators and relations, and show that they are isomorphic. We call the common resulting algebra an ADU algebra. For each ADU algebra we display a $\mathbb{Z}$-grading and a linear basis. We also show that the center is isomorphic to a polynomial algebra in two variables. We obtain ADU algebra modules from each of the above seven examples of uniform posets.

We have a remark about the place of down-up algebras and ADU algebras in ring theory. A down-up algebra can be viewed as an ambiskew polynomial ring [7, Section 3], which in turn can be viewed as a generalized Weyl algebra [1], [7, Prop. 2.1]. By a comment in [8, p. 48] that cites a preprint version of the present paper, an ADU algebra can also be viewed in this way. Hoping to keep our paper accessible to nonexperts in ring theory, we will avoid this point of view and use only linear algebra.

Recall the natural numbers $\mathbb{N}=\{0,1,2, \ldots\}$ and integers $\mathbb{Z}=\{0, \pm 1, \pm 2, \ldots\}$.

\section{Augmented down-up algebras}

Our conventions for the paper are as follows. An algebra is meant to be associative and have a 1 . A subalgebra has the same 1 as the parent algebra. Let $\mathbb{F}$ denote a field. Let $\lambda$ denote an indeterminate. Let $\mathbb{F}\left[\lambda, \lambda^{-1}\right]$ denote the $\mathbb{F}$-algebra of Laurent polynomials in $\lambda$ that have all coefficients in $\mathbb{F}$. Pick $\psi \in \mathbb{F}\left[\lambda, \lambda^{-1}\right]$ and write $\psi=\sum_{i \in \mathbb{Z}} \alpha_{i} \lambda^{i}$. By the support of $\psi$ we mean the set $\left\{i \in \mathbb{Z} \mid \alpha_{i} \neq 0\right\}$. This set is finite.

Fix distinct $s, t \in \mathbb{Z}$. Define

$$
\mathbb{F}\left[\lambda, \lambda^{-1}\right]_{s, t}=\operatorname{Span}\left\{\lambda^{i} \mid i \in \mathbb{Z}, i \neq s, i \neq t\right\} .
$$


Note that

$$
\mathbb{F}\left[\lambda, \lambda^{-1}\right]=\mathbb{F}\left[\lambda, \lambda^{-1}\right]_{s, t}+\mathbb{F} \lambda^{s}+\mathbb{F} \lambda^{t} \quad \text { (direct sum) }
$$

For $\psi \in \mathbb{F}\left[\lambda, \lambda^{-1}\right]$ the following are equivalent: (i) $\psi \in \mathbb{F}\left[\lambda, \lambda^{-1}\right]_{s, t}$; (ii) the integers $s, t$ are not in the support of $\psi$.

Fix a nonzero $q \in \mathbb{F}$ that is not a root of unity.

Definition 2.1. For $\varphi \in \mathbb{F}\left[\lambda, \lambda^{-1}\right]_{s, t}$ the $\mathbb{F}$-algebra $\mathbb{A}=\mathbb{A}_{q}(s, t, \varphi)$ has generators $e, f$, $k^{ \pm 1}$ and relations

$$
\begin{aligned}
& k k^{-1}=1, \quad k^{-1} k=1, \\
& k e=q^{2} e k, \quad k f=q^{-2} f k, \\
& e^{2} f-\left(q^{-2 s}+q^{-2 t}\right) e f e+q^{-2 s-2 t} f e^{2}=e \varphi(k), \\
& e f^{2}-\left(q^{-2 s}+q^{-2 t}\right) f e f+q^{-2 s-2 t} f^{2} e=\varphi(k) f .
\end{aligned}
$$

Remark 2.2. Referring to Definition 2.1, consider the special case in which $\varphi \in \mathbb{F}$. Then the relations (2.1), (2.2) become the defining relations for the down-up algebra $A\left(q^{-2 s}+\right.$ $\left.q^{-2 t},-q^{-2 s-2 t}, \varphi\right)$.

Definition 2.3. For $\phi \in \mathbb{F}\left[\lambda, \lambda^{-1}\right]_{s, t}$ the $\mathbb{F}$-algebra $\mathbb{B}=\mathbb{B}_{q}(s, t, \phi)$ has generators $C_{s}, C_{t}$, $E, F, K^{ \pm 1}$ and relations

$$
\begin{aligned}
& C_{s}, C_{t} \quad \text { are central, } \\
& K K^{-1}=1, \quad K^{-1} K=1, \\
& K E=q^{2} E K, \quad K F=q^{-2} F K, \\
& F E=C_{s} q^{s} K^{s}+C_{t} q^{t} K^{t}+\phi(q K), \\
& E F=C_{s} q^{-s} K^{s}+C_{t} q^{-t} K^{t}+\phi\left(q^{-1} K\right) .
\end{aligned}
$$

Next we describe how the algebras in Definition 2.1 and Definition 2.3 are related.

Definition 2.4. We define an $\mathbb{F}$-linear map $\mathbb{F}\left[\lambda, \lambda^{-1}\right] \rightarrow \mathbb{F}\left[\lambda, \lambda^{-1}\right], \psi \mapsto \psi_{s, t}$ as follows. For $\psi \in \mathbb{F}\left[\lambda, \lambda^{-1}\right]$,

$$
\psi_{s, t}(\lambda)=\psi\left(q^{-1} \lambda\right)-\left(q^{-2 s}+q^{-2 t}\right) \psi(q \lambda)+q^{-2 s-2 t} \psi\left(q^{3} \lambda\right) .
$$

Recall the basis $\left\{\lambda^{i}\right\}_{i \in \mathbb{Z}}$ for $\mathbb{F}\left[\lambda, \lambda^{-1}\right]$.

Lemma 2.5. Consider the map $\psi \mapsto \psi_{s, t}$ from Definition 2.4. For $i \in \mathbb{Z}$ the vector $\lambda^{i}$ is an eigenvector for the map. The corresponding eigenvalue is $q^{3 i}\left(q^{-2 i}-q^{-2 s}\right)\left(q^{-2 i}-q^{-2 t}\right)$. This eigenvalue is zero if and only if $i \in\{s, t\}$.

Proof. Use Definition 2.4.

The following two lemmas are routine consequences of Lemma 2.5.

Lemma 2.6. For the map $\psi \mapsto \psi_{s, t}$ from Definition 2.4 the image is $\mathbb{F}\left[\lambda, \lambda^{-1}\right]_{s, t}$ and the kernel is $\mathbb{F} \lambda^{s}+\mathbb{F} \lambda^{t}$. 
Lemma 2.7. For the map $\psi \mapsto \psi_{s, t}$ from Definition 2.4 the restriction to $\mathbb{F}\left[\lambda, \lambda^{-1}\right]_{s, t}$ is invertible.

Let $\varphi, \phi \in \mathbb{F}\left[\lambda, \lambda^{-1}\right]_{s, t}$ such that $\varphi=\phi_{s, t}$. We are going to show that the algebras $\mathbb{A}_{q}(s, t, \varphi)$ and $\mathbb{B}_{q}(s, t, \phi)$ are isomorphic.

Lemma 2.8. For $\phi \in \mathbb{F}\left[\lambda, \lambda^{-1}\right]_{s, t}$ the following hold in $\mathbb{B}_{q}(s, t, \phi)$ :

$$
\begin{aligned}
& C_{s}=\frac{q^{-t} F E-q^{t} E F+q^{t} \phi\left(q^{-1} K\right)-q^{-t} \phi(q K)}{q^{s-t}-q^{t-s}} K^{-s}, \\
& C_{t}=\frac{q^{-s} F E-q^{s} E F+q^{s} \phi\left(q^{-1} K\right)-q^{-s} \phi(q K)}{q^{t-s}-q^{s-t}} K^{-t} .
\end{aligned}
$$

Moreover the algebra $\mathbb{B}_{q}(s, t, \phi)$ is generated by $E, F, K^{ \pm 1}$.

Proof. We first verify (2.5). In the expression on the right in (2.5), eliminate $F E$ and $E F$ using (2.3) and (2.4). After a routine simplification (2.5) is verified. The equation (2.6) is similarly verified. The last assertion follows from (2.5), (2.6).

Lemma 2.9. For $\phi \in \mathbb{F}\left[\lambda, \lambda^{-1}\right]_{s, t}$ the following hold in $\mathbb{B}_{q}(s, t, \phi)$ :

$$
\begin{aligned}
& E^{2} F-\left(q^{-2 s}+q^{-2 t}\right) E F E+q^{-2 s-2 t} F E^{2}=E \varphi(K), \\
& E F^{2}-\left(q^{-2 s}+q^{-2 t}\right) F E F+q^{-2 s-2 t} F^{2} E=\varphi(K) F .
\end{aligned}
$$

In the above lines $\varphi=\phi_{s, t}$.

Proof. We first verify (2.7). In the expression on the left in (2.7), view $E^{2} F=E(E F)$, $E F E=E(F E), F E^{2}=(F E) E$ and eliminate each parenthetical expression using (2.3) and (2.4). Simplify the result using $K E=q^{2} E K$ along with $\varphi=\phi_{s, t}$ and Definition 2.4. The equation (2.7) is now verified. The equation (2.8) is similarly verified.

The following definition is motivated by Lemma 2.8 .

Definition 2.10. For $\varphi \in \mathbb{F}\left[\lambda, \lambda^{-1}\right]_{s, t}$ let $c_{s}, c_{t}$ denote the following elements in $\mathbb{A}_{q}(s, t$, $\varphi)$ :

$$
\begin{aligned}
& c_{s}=\frac{q^{-t} f e-q^{t} e f+q^{t} \phi\left(q^{-1} k\right)-q^{-t} \phi(q k)}{q^{s-t}-q^{t-s}} k^{-s}, \\
& c_{t}=\frac{q^{-s} f e-q^{s} e f+q^{s} \phi\left(q^{-1} k\right)-q^{-s} \phi(q k)}{q^{t-s}-q^{s-t}} k^{-t} .
\end{aligned}
$$

In the above lines $\phi$ denotes the unique element in $\mathbb{F}\left[\lambda, \lambda^{-1}\right]_{s, t}$ such that $\varphi=\phi_{s, t}$.

Lemma 2.11. With the notation and assumptions of Definition 2.10, the elements $c_{s}, c_{t}$ are central in $\mathbb{A}_{q}(s, t, \varphi)$. Moreover

$$
\begin{aligned}
& f e=c_{s} q^{s} k^{s}+c_{t} q^{t} k^{t}+\phi(q k), \\
& e f=c_{s} q^{-s} k^{s}+c_{t} q^{-t} k^{t}+\phi\left(q^{-1} k\right)
\end{aligned}
$$


Proof. We first show that $c_{s}$ is central in $\mathbb{A}_{q}(s, t, \varphi)$. To do this we show $c_{s} e=e c_{s}$, $c_{s} f=f c_{s}, c_{s} k=k c_{s}$. To verify these equations, eliminate each occurrence of $c_{s}$ using (2.9), and simplify the result using the relations in Definition 2.1. We have shown that $c_{s}$ is central in $\mathbb{A}_{q}(s, t, \varphi)$. One similarly shows that $c_{t}$ is central in $\mathbb{A}_{q}(s, t, \varphi)$. We now verify (2.11). In the expression on the right in (2.11), eliminate $c_{s}, c_{t}$ using (2.9), (2.10). After a routine simplification (2.11) is verified. The equation (2.12) is similarly verified.

Theorem 2.12. Given $\varphi, \phi \in \mathbb{F}\left[\lambda, \lambda^{-1}\right]_{s, t}$ such that $\varphi=\phi_{s, t}$. Then there exists an $\mathbb{F}$ algebra isomorphism $\mathbb{A}_{q}(s, t, \varphi) \rightarrow \mathbb{B}_{q}(s, t, \phi)$ that sends

$$
e \mapsto E, \quad f \mapsto F, \quad k^{ \pm 1} \mapsto K^{ \pm 1} .
$$

The inverse isomorphism sends

$$
C_{s} \mapsto c_{s}, \quad C_{t} \mapsto c_{t}, \quad E \mapsto e, \quad F \mapsto f, \quad K^{ \pm 1} \mapsto k^{ \pm 1}
$$

where $c_{s}, c_{t}$ are from Definition 2.10.

Proof. Combine Lemmas 2.8, 2.9, 2.11.

Definition 2.13. By an augmented down-up algebra we mean an algebra $\mathbb{A}_{q}(s, t, \varphi)$ from Definition 2.1 or an algebra $\mathbb{B}_{q}(s, t, \phi)$ from Definition 2.3.

Consider the algebra $\mathbb{B}=\mathbb{B}_{q}(s, t, \phi)$ from Definition 2.3. In Section 3 we are going to show that the elements $C_{s}, C_{t}$ generate the center $Z(\mathbb{B})$, and that $Z(\mathbb{B})$ is isomorphic to a polynomial algebra in two variables. Because of this and following [6, p. 27], it seems appropriate to call $C_{s}, C_{t}$ the Casimir elements for $\mathbb{B}_{q}(s, t, \phi)$.

\section{A $\mathbb{Z}$-grading and linear basis for $\mathbb{B}_{q}(s, t, \phi)$}

Recall the algebra $\mathbb{B}=\mathbb{B}_{q}(s, t, \phi)$ from Definition 2.3. In this section we display a $\mathbb{Z}$ grading for $\mathbb{B}$. We also display a basis for the $\mathbb{F}$-vector space $\mathbb{B}$.

Let $\mathcal{A}$ denote an $\mathbb{F}$-algebra. By a $\mathbb{Z}$-grading of $\mathcal{A}$ we mean a sequence $\left\{\mathcal{A}_{n}\right\}_{n \in \mathbb{Z}}$ consisting of subspaces of $\mathcal{A}$ such that

$$
\mathcal{A}=\sum_{n \in \mathbb{Z}} \mathcal{A}_{n} \quad \text { (direct sum) }
$$

and $\mathcal{A}_{m} \mathcal{A}_{n} \subseteq \mathcal{A}_{m+n}$ for all $m, n \in \mathbb{Z}$. Let $\left\{\mathcal{A}_{n}\right\}_{n \in \mathbb{Z}}$ denote a $\mathbb{Z}$-grading of $\mathcal{A}$. For $n \in \mathbb{Z}$ we call $\mathcal{A}_{n}$ the $n$-homogeneous component of $\mathcal{A}$. We refer to $n$ as the degree of $\mathcal{A}_{n}$. An element of $\mathcal{A}$ is said to be homogeneous of degree $n$ whenever it is contained in $\mathcal{A}_{n}$.

Theorem 3.1. The algebra $\mathbb{B}$ has a $\mathbb{Z}$-grading $\left\{\mathbb{B}_{n}\right\}_{n \in \mathbb{Z}}$ with the following properties:

(i) The $\mathbb{F}$-vector space $\mathbb{B}_{0}$ has a basis

$$
K^{h} C_{s}^{i} C_{t}^{j} \quad h \in \mathbb{Z}, \quad i, j \in \mathbb{N} .
$$

(ii) For $n \geq 1$, the $\mathbb{F}$-vector space $\mathbb{B}_{n}$ has a basis

$$
F^{n} K^{h} C_{s}^{i} C_{t}^{j} \quad h \in \mathbb{Z}, \quad i, j \in \mathbb{N} .
$$


(iii) For $n \geq 1$, the $\mathbb{F}$-vector space $\mathbb{B}_{-n}$ has a basis

$$
E^{n} K^{h} C_{s}^{i} C_{t}^{j} \quad h \in \mathbb{Z}, \quad i, j \in \mathbb{N} .
$$

Moreover the union of (3.1)-(3.3) is a basis for the $\mathbb{F}$-vector space $\mathbb{B}$.

Proof. Routinely applying the Bergman diamond lemma [3, Theorem 1.2] one finds that the union of (3.1)-(3.3) is a basis for the $\mathbb{F}$-vector space $\mathbb{B}$. Let $\mathbb{B}_{0}$ denote the subspace of $\mathbb{B}$ spanned by (3.1). For $n \geq 1$ let $\mathbb{B}_{n}$ and $\mathbb{B}_{-n}$ denote the subspaces of $\mathbb{B}$ spanned by (3.2) and (3.3), respectively. We show that $\left\{\mathbb{B}_{n}\right\}_{n \in \mathbb{Z}}$ is a $\mathbb{Z}$-grading of $\mathbb{B}$. By construction the sum $\mathbb{B}=\sum_{n \in \mathbb{Z}} \mathbb{B}_{n}$ is direct. By construction and since $C_{s}, C_{t}$ are central we have $C_{s} \mathbb{B}_{n} \subseteq \mathbb{B}_{n}$ and $C_{t} \mathbb{B}_{n} \subseteq \mathbb{B}_{n}$ for $n \in \mathbb{Z}$. Using $K E=q^{2} E K$ and $K F=q^{-2} F K$ we find $K^{ \pm 1} \mathbb{B}_{n} \subseteq \mathbb{B}_{n}$ for $n \in \mathbb{Z}$. Using (2.3) and (2.4) we find $E \mathbb{B}_{n} \subseteq \mathbb{B}_{n-1}$ and $F \mathbb{B}_{n} \subseteq \mathbb{B}_{n+1}$ for $n \in \mathbb{Z}$. By these comments and the construction we see that $\mathbb{B}_{m} \mathbb{B}_{n} \subseteq \mathbb{B}_{m+n}$ for all $m, n \in \mathbb{Z}$. Therefore $\left\{\mathbb{B}_{n}\right\} \in \mathbb{Z}$ is a $\mathbb{Z}$-grading of $\mathbb{B}$. The result follows.

We emphasize a few points from Theorem 3.1.

Corollary 3.2. With respect to the above $\mathbb{Z}$-grading of $\mathbb{B}$, the generators $C_{s}, C_{t}, E, F, K^{ \pm 1}$ are homogeneous with the following degrees:

\begin{tabular}{c|ccccc}
$v$ & $C_{s}$ & $C_{t}$ & $E$ & $F$ & $K^{ \pm 1}$ \\
\hline degree of $v$ & 0 & 0 & -1 & 1 & 0
\end{tabular}

Corollary 3.3. The homogeneous component $\mathbb{B}_{0}$ is the subalgebra of $\mathbb{B}$ generated by $C_{s}, C_{t}, K^{ \pm 1}$. The algebra $\mathbb{B}_{0}$ is commutative.

Let $\left\{\lambda_{i}\right\}_{i=0}^{2}$ denote mutually commuting indeterminates.

Corollary 3.4. There exists an $\mathbb{F}$-algebra isomorphism $\mathbb{B}_{0} \rightarrow \mathbb{F}\left[\lambda_{0}^{ \pm 1}, \lambda_{1}, \lambda_{2}\right]$ that sends

$$
K^{ \pm 1} \mapsto \lambda_{0}^{ \pm 1}, \quad C_{s} \mapsto \lambda_{1}, \quad C_{t} \mapsto \lambda_{2}
$$

The $\mathbb{Z}$-grading $\left\{\mathbb{B}_{n}\right\}_{n \in \mathbb{Z}}$ has the following interpretation.

Lemma 3.5. Consider the $\mathbb{F}$-linear map $\mathbb{B} \rightarrow \mathbb{B}, \xi \mapsto K^{-1} \xi K$. For $n \in \mathbb{Z}$ the $n$ homogeneous component $\mathbb{B}_{n}$ is an eigenspace of this map. The corresponding eigenvalue is $q^{2 n}$.

Proof. Use the basis for $\mathbb{B}_{n}$ given in Theorem 3.1, along with the relations $K E=q^{2} E K$ and $K F=q^{-2} F K$.

Corollary 3.6. The homogeneous component $\mathbb{B}_{0}$ consists of the elements in $\mathbb{B}$ that commute with $K$.

Proof. Immediate from Lemma 3.5. 


\section{The center of $\mathbb{B}_{q}(s, t, \phi)$}

Recall the algebra $\mathbb{B}=\mathbb{B}_{q}(s, t, \phi)$ from Definition 2.3. In this section we describe the center $Z(\mathbb{B})$.

Theorem 4.1. The following is a basis for the $\mathbb{F}$-vector space $Z(\mathbb{B})$ :

$$
C_{s}^{i} C_{t}^{j} \quad i, j \in \mathbb{N} .
$$

Proof. By Theorem 3.1 the elements (4.1) are linearly independent over $\mathbb{F}$, so they form a basis for a subspace of $\mathbb{B}$ which we denote by $Z^{\prime}$. We show $Z^{\prime}=Z(\mathbb{B})$. The elements $C_{s}, C_{t}$ are central in $\mathbb{B}$ so $Z^{\prime} \subseteq Z(\mathbb{B})$. To obtain the reverse inclusion, pick $\xi \in Z(\mathbb{B})$. The element $\xi$ commutes with $K$, so $\xi \in \mathbb{B}_{0}$ by Corollary 3.6. Recall the basis (3.1) for $\mathbb{B}_{0}$. Writing $\xi$ in this basis, we find $\xi=\sum_{h \in \mathbb{Z}} K^{h} \xi_{h}$ where $\xi_{h} \in Z^{\prime}$ for $h \in \mathbb{Z}$. Using $K E=q^{2} E K$ and $\xi E=E \xi$ we obtain $0=E \sum_{h \in \mathbb{Z}} K^{h} \xi_{h}\left(q^{2 h}-1\right)$. Combining this with Theorem 3.1 we find $\xi_{h}=0$ for all nonzero $h \in \mathbb{Z}$. Therefore $\xi=\xi_{0} \in Z^{\prime}$. We have shown $Z^{\prime}=Z(\mathbb{B})$ and the result follows.

Corollary 4.2. There exists an $\mathbb{F}$-algebra isomorphism $Z(\mathbb{B}) \rightarrow \mathbb{F}\left[\lambda_{1}, \lambda_{2}\right]$ that sends

$$
C_{s} \mapsto \lambda_{1}, \quad C_{t} \mapsto \lambda_{2}
$$

\section{Uniform posets}

Recall the algebras $\mathbb{A}_{q}(s, t, \varphi)$ from Definition 2.1. In this section we discuss how these algebras are related to the uniform posets [10].

Throughout this section we assume that $\mathbb{F}$ is the complex number field $\mathbb{C}$. Let $P$ denote a finite ranked poset with fibers $\left\{P_{i}\right\}_{i=0}^{N}[10$, p. 194]. Let $\mathbb{C} P$ denote the vector space over $\mathbb{C}$ with basis $P$. Let $\operatorname{End}(\mathbb{C} P)$ denote the $\mathbb{C}$-algebra consisting of all $\mathbb{C}$-linear maps from $\mathbb{C} P$ to $\mathbb{C} P$. We now define three elements in $\operatorname{End}(\mathbb{C} P)$ called the lowering, raising, and $q$-rank operators. For $x \in P$, the lowering operator sends $x$ to the sum of the elements in $P$ that are covered by $x$. The raising operator sends $x$ to the sum of the elements in $P$ that cover $x$. The $q$-rank operator sends $x$ to $q^{N-2 i} x$ where $x \in P_{i}$.

In [10] we introduced a class of finite ranked posets said to be uniform. We refer the reader to that article for a detailed description of these posets. See also [2, p. 306] and [9], [11]. In [10, Section 3] we gave eleven examples of uniform posets. We are going to show that six of these examples give an $\mathbb{A}_{q}(s, t, \varphi)$-module. These six examples are listed in the first six rows of the table below. The remaining row of the table contains an example $\operatorname{Polar}_{b}^{\text {top }}(N, \epsilon)$ which is defined as follows. Start with the poset $\operatorname{Polar}_{b}(N, \epsilon)$ which we denote by $P$. Using $P$ we define an undirected graph $\Gamma$ as follows. The vertex set of $\Gamma$ consists of the top fiber $P_{N}$ of $P$. Vertices $y, z \in P_{N}$ are adjacent in $\Gamma$ whenever they are distinct and cover a common element of $P$. The graph $\Gamma$ is often called a dual polar graph [4, p. 274], [12, Section 16]. Fix a vertex $x \in P_{N}$. Using $x$ we define a partial order $\leq$ on $P_{N}$ as follows. For $y, z \in P_{N}$ let $y \leq z$ whenever $\partial(x, y)+\partial(y, z)=\partial(x, z)$, where $\partial$ denotes path-length distance in $\Gamma$. We have turned $P_{N}$ into a poset. We call this poset $\operatorname{Polar}_{b}^{\mathrm{top}}(N, \epsilon)$. Using [12, Proposition 26.4] one checks that $\operatorname{Polar}_{b}^{\mathrm{top}}(N, \epsilon)$ is uniform.

Theorem 5.1. In each row of the table below we give an example of a uniform poset $P$. For each example we display integers $s<t$ and a Laurent polynomial $\varphi \in \mathbb{F}\left[\lambda, \lambda^{-1}\right]_{s, t}$. 
In each case the vector space $\mathbb{C} P$ becomes an $\mathbb{A}_{q}(s, t, \varphi)$-module such that the generator e (resp. f) (resp. $k$ ) acts on $\mathbb{C} P$ as the lowering (resp. raising) (resp. q-rank) operator for $P$. For convenience, for each example we display the element $\phi \in \mathbb{F}\left[\lambda, \lambda^{-1}\right]_{s, t}$ such that $\varphi=\phi_{s, t}$.

\begin{tabular}{c|cccc} 
example & $s$ & $t$ & $\varphi$ & $\phi$ \\
\hline $\operatorname{Polar}_{b}(N, \epsilon)$ & 0 & 1 & $-\left(q+q^{-1}\right)\left(q^{2 N+1+2 \epsilon} \lambda^{2}+q^{N-3} \lambda^{-1}\right)$ & $-\frac{q^{2 N+2 \epsilon} \lambda^{2}+q^{N-1} \lambda^{-1}}{\left(q-q^{-1}\right)^{2}}$ \\
$\mathrm{~A}_{b}(N, M)$ & -1 & 0 & $-\left(q+q^{-1}\right) q^{N+2 M+1} \lambda$ & $-\frac{q^{N+2 M-1}}{\left(q-q^{-1}\right)^{2}} \lambda$ \\
$\operatorname{Alt}_{b}(N)$ & -2 & -1 & $-\left(q+q^{-1}\right) q^{2 N+1}$ & $-\frac{q^{2 N-2}}{\left(q-q^{-1}\right)^{2}}$ \\
$\operatorname{Her}_{q}(N)$ & -2 & -1 & $-\left(q+q^{-1}\right) q^{2 N+2}$ & $-\frac{q^{2 N-1}}{\left(q-q^{-1}\right)^{2}}$ \\
$\operatorname{Quad}_{b}(N)$ & -2 & -1 & $-\left(q+q^{-1}\right) q^{2 N+3}$ & $-\frac{q^{2 N}}{\left(q-q^{-1}\right)^{2}}$ \\
$\operatorname{Hem}_{b}(N)$ & -2 & -1 & $-\left(q+q^{-1}\right) q^{2 N+1}$ & $-\frac{q^{2 N-2}}{\left(q-q^{-1}\right)^{2}}$ \\
$\operatorname{Polar}_{b}^{\operatorname{top}}(N, \epsilon)$ & -2 & -1 & $-\left(q+q^{-1}\right) q^{2 N+3+2 \epsilon}$ & $-\frac{q^{2 N+2 \epsilon}}{\left(q-q^{-1}\right)^{2}}$
\end{tabular}

In the above table $b=q^{2}$.

Proof. For each example except the last, our assertions follow routinely from [10, Theorem 3.2]. For the last example $\operatorname{Polar}_{b}^{\text {top }}(N, \epsilon)$ our assertions follow from [12, Theorem 1.10]. Note that the parameter denoted $\epsilon$ in [12, Theorem 1.10] is one more than the parameter denoted $\epsilon$ in [10, p. 201].

\section{Acknowledgement}

The main inspiration for the ADU algebra concept comes from the second author's thesis [12] concerning the uniform poset $\operatorname{Polar}_{b}^{\text {top }}(N, \epsilon)$. To be more precise, it was his discovery of two central elements that he called $C_{1}, C_{2}$ [12, Section 28] that suggested to us how to define an ADU algebra.

\section{References}

[1] V. Bavula and D. A. Jordan, Isomorphism problems and groups of automorphisms for generalized Weyl algebras, Trans. Amer. Math. Soc. 353 (2001), 769-794.

[2] G. Benkart and T. Roby, Down-up algebras, J. Algebra 209 (1998), 305-344.

[3] G. Bergman, The diamond lemma for ring theory, Adv. Math. 29 (1978), 178-218.

[4] A. E. Brouwer, A. M. Cohen, and A. Neumaier, Distance-Regular Graphs, Springer-Verlag, Berlin, 1989.

[5] T. Cassidy and B. Shelton, Basic properties of generalized down-up algebras, J. Algebra 279 (2004), 402-421. 
[6] J. C. Jantzen, Lectures on quantum groups, Graduate Studies in Mathematics 6, Amer. Math. Soc., Providence RI, 1996.

[7] D. A. Jordan, Down-up algebras and ambiskew polynomial rings, J. Algebra 228 (2000), 311346.

[8] D. A. Jordan and I. Wells, Simple ambiskew polynomial rings, J. Algebra 382 (2013), 46-70.

[9] S. Miklavic and P. Terwilliger, Bipartite $Q$-polynomial distance-regular graphs and uniform posets, J. Algebraic Combin. 38 (2013), 225-242.

[10] P. Terwilliger, The incidence algebra of a uniform poset, in: D. Ray-Chaudhuri (ed.), Coding theory and design theory, Part I, IMA Vol. Math. Appl., 20, Springer, New York, 1990, 193212.

[11] P. Terwilliger, Quantum matroids, in: E. Bannai and A. Munemasa (eds.), Progress in algebraic combinatorics (Fukuoka, 1993), Adv. Stud. Pure Math., 24, Math. Soc. Japan, Tokyo, 1996, 323-441.

[12] C. Worawannotai, Dual polar graphs, the quantum algebra $U_{q}\left(\mathfrak{s l}_{2}\right)$, and Leonard systems of dual $q$-Krawtchouk type, Linear Algebra Appl. 438 (2013), 443-497. 